\title{
TECHNOLOGY OF MulTi-Agent CONTROL For INDUSTRIAL Automation With logical Processing OF CONTRADICTIONS
}

\author{
Valentin E. Pryanichnikov, Aleksander A. Aryskin, Stanislav R. Eprikov, Kirill B. \\ Kirsanov, Roman V. Khelemendik, Aleksander Ya. Ksenzenko, \\ Evgeny A. Prysev \& Aleksander S. Travushkin
}
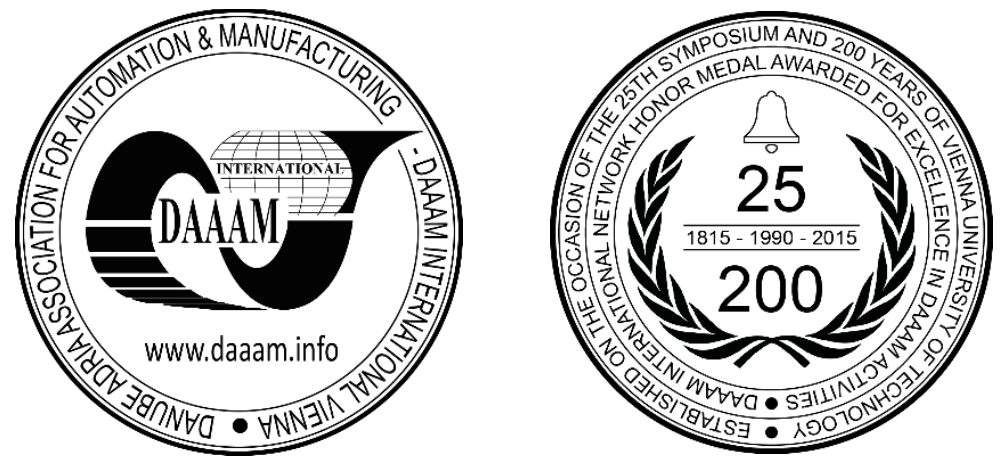

This Publication has to be referred as: V[alentin] E. Pryanichnikov, A[leksander] A. Aryskin, S[tanislav] R. Eprikov, K[irill] B. Kirsanov, R[oman] V. Khelemendik, A[leksander] Ya. Ksenzenko, E[vgeny] A. Prysev \& A[leksander] S. Travushkin (2017). Technology of Multi-Agent Control for Industrial Automation with Logical Processing of Contradictions, Proceedings of the 28th DAAAM International Symposium, pp.1202-1207, B. Katalinic (Ed.), Published by DAAAM International, ISBN 978-3-902734-11-2, ISSN 1726-9679, Vienna, Austria DOI: $10.2507 / 28$ th.daaam.proceedings. 167

\begin{abstract}
The paper related to the problem of creation the new technology of multi-agent control for industrial automation - creating the automated system, realising multi-recipes production of a large number of types with logical processing of contradictions. This required to find a proper software decisions for distant access, reprogramming and monitoring the production line even during it's functioning. The created system of logical processing, basing on brunching time logics, and parallel software, controlling production line as well as the imitation modelling, are a new way for intelligent industry 4.0 .
\end{abstract}

Keywords: industry 4.0; intelligent manufacturing; sensor systems; cyber-physical systems; Internet of Things; logical contradictions; industrial automation

\section{Introduction}

Technological advances had significantly, rapidly increase the productivity last time. To describe shot that global industrial revolutions I-IV, we can use the one-word characteristics: mechanization - electrification - automation intellectualization. The basis for the concept of Industry 4.0 are: "Internet of Things", "Big Data", "Cyber-physical system". Under this concept, one of the main tasks is to ensure effective releasing of even minimal parties out-coming production with the rapidly changing structure of the production lines. Using built-in sensors/actuator network access leads to the necessity of creating new methods of optimisation for resource conservation in a production environment. It was necessary to create a mechanism for predicting the faults of the components or equipment, as well as the possibility of integrating the process of self-repair, finding alternative solutions to current production problems, the resolution of the relevant repair or maintenance. 
If more recently, consumers of automation did not provide the manufacturer access to the purchased equipment. In recent years, it is appeared a large demand for life support equipment. One of the leaders in implementation of this concept is the concern FESTO AG, working in more, than hundred countries with 300,000 users and in practice, providing justin-time production.

In the Russian Federation, not all businesses are ready to introduce the concept of "Smart production", as this is a difficult, long and expensive process. At the same time enterprise, created "from scratch", more receptive to such innovations. International laboratory "Sensorika", together with specialists and graduate students from MSTU "Stankin", INET RSUH, KIAM Russian Academy of sciences, JSC “TechInvest” has developed a technology for implementation and deployment of "Smart production" by building a new automatic line for manufacturing the foam-glass and products from it in the city Kimry. The system automation implemented in the framework of this project, is based on the previously patented methods for automatically resolve logical contradictions (refined to adapt to the needs and tasks). This technique allows to predict the probable failures of the system in certain situations and is one of the foundations of the reliability of the project. Automation implemented into the project is a symbiosis of decentralised systems integrated into a digital network with the possibility of switching to automated program or to manual control, if necessary. The developed concept allows to perform non-trivial tasks for the intelligent control of executive elements of technological process, for example, switching valves, dampers, motor control for input/output, for moving components in the production lines according with the flexible manufacturing process. In this process are involved specialised transport system, developed with the participation of the International laboratory "Sensorika". It is used for drying and moving the produced details and finished products in the production space about $1400 \mathrm{~m} 2$. This system is made on the basis of individual robotic trucks, which are driven by electro engines and a set of sensor devices. One such transport unit is able to move along a complicated paths the internal elements and finished products, weighing up to 2-3 tons in hard conditions with high humidity. Part of the overall management system of the production cycle is the interface, that provides access to it with the remote monitoring, testing and upgrading processes. Developed control system for transport and manipulation robotic devices, initially as a set of peripheral devices, which don't have a characteristics of flexibility in our project. At the same time it provides "intelligent data entry" through a system of sensors and feedback lines, controlling the operation of all actuators with logical analysis and the detection of incorrect manufacturing processes, which significantly reduces the probability of violation of the technological cycle and emergency. The listed requirements to the modern production, that we are implementing, to some extent fits into the concept of the next industrial revolution, called "Industry 4.0".

\section{Experience in implementing the flexible production line.}

The control system ensures fully automatic operation of the production, realised in two separate parts of the workshop. In one of them the preparation of a sodium glass substance with the appropriate additives is made, in the another, it is handled by shape, drying, final processing and packaging as the finished product, followed by transportation system for shipment to the consumer or to the warehouse of finished products.

The technological scheme for preparing the substance is shown in Fig. 1. It's work is formalised/described with around 200 logical variables. In reactor 1-3 is operating with large capacity, the main components of the substance are mixed in the required proportions with observance of the temperature regime. At the initial stage, this process requires the initial forced heating of the mixture to a temperature above 100 degrees. For this purpose, a special steam generator with a capacity of $250 \mathrm{~kg} / \mathrm{h}$ with a steam temperature up to160 degrees is present in the circuit. The prepared mixture enters the storage tanks stock. During the mixing process, an exothermic chemical reaction develops, which requires forced cooling to a temperature of less degrees by means of a heat exchanger. For its' functioning, tanks with a reserve of cold and hot water are provided. The cooled mixture enters the vessels/tanks, after which it is envisaged to add a special fiberglass suspension to it from the containers, which provides the required consumer characteristics of the future product.

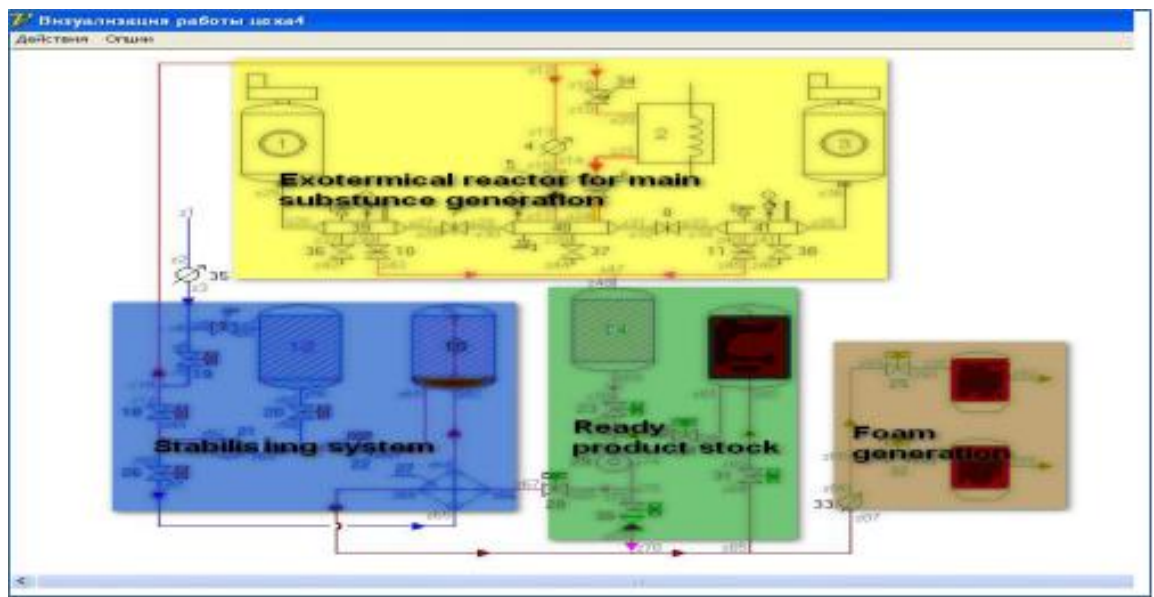

Fig. 1. The graphical interface of the simulation program - technological scheme with logical control of failures. 
All valves have remote control mechanisms. The executive and regulating elements of the technological scheme are equipped with microprocessors for controlling the execution of control commands. The temperature regime, the level of filling of tanks and the pressure in them is controlled by means of suitable sensors. At the most critical nodes of the technological scheme, duplication of monitoring sensors is applied. The system ensures the execution of the required opening and closing time intervals, valves through which the components are fed. The developed control concept allows performing complex tasks of influence on the actuating elements in the process flow diagram. The preparation of each recipe for the preparation of a substance with the tracking time intervals for further execution of the control program is initially performed by the plant's technologist. After preparing the pilot batch of the product and passing the quality control, a decision is taken to write the recipe into the database. Then, this recipe can be called up by the operator on duty and executed without the help of a technologist.

Spillage of the finished substance with added components is made in the drying-filling part of the shop, Fig. 2. Its technological scheme is based on the use of transport trolleys, driven by gear racks, which, in turn, are moved by pairs of electric motors with a power of $1.5 \mathrm{~kW}$ through retarding reducers. The operational information support of the control system of this shop and the control of the execution of the specified trajectory of moving the carriages is provided by a set of induction and optical sensors, as well as a video monitoring system. A special additional system avoids the collision of loaded and empty trolleys.

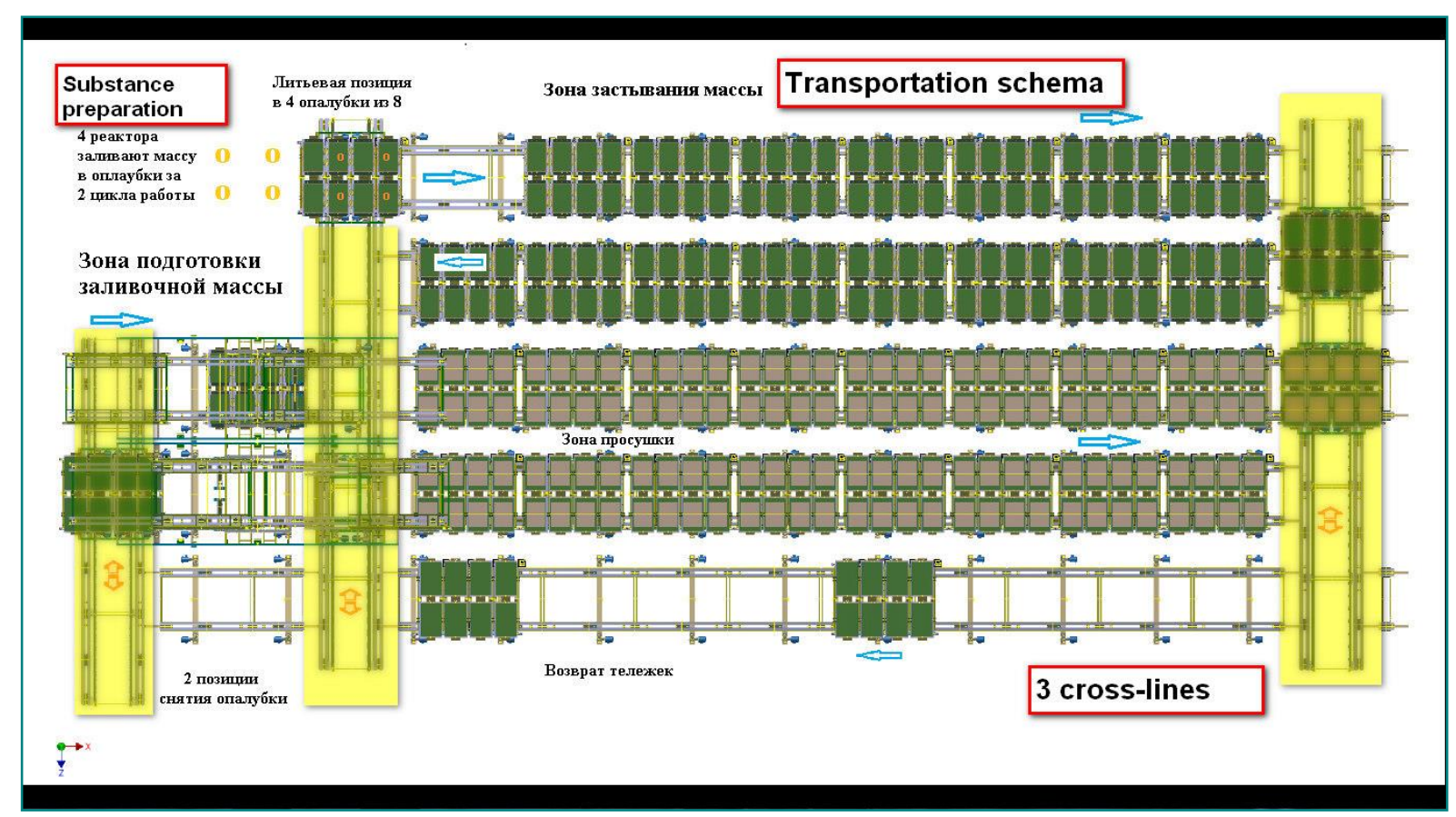

Fig. 2. Diagram of the reconfigurable transport system (fragment of drying-packing workshop).

The automatic control system of the actuating elements is located in the immediate vicinity of the workshop in a room protected by a glass partition. There is also a manual control panel and a duty operator's workplace.

The control system is a symbiosis of decentralised systems, combined into a common digital network with the possibility of switching to hard software or manual control, if necessary.

\section{Development of methods of heuristic deduction for the representation of technological knowledge.}

The development of the control system (basing on the experts-technologists knowledge) was implemented, using our IGEC technology, presented in [1-4]. IGEC technology is a combination of four main directions of mathematical approaches conventionally called Ancient Greek, Ancient Egyptian, Ancient Indian and Ancient Chinese. This allows to use its strengths for each direction, and to supplement and harmonise the results obtained, for example, to eliminate contradictions (conflicts) arising from different traditions (approaches). This technology has been successfully used, for example, in the development of the basic software and hardware elements of the network of associative laboratories and operational centres in the framework of the project "Intellectual Robotronics", initiated by the Central European Branch of the International Engineering Academy in cooperation with the universities of Russia, Croatia and Austria. With the use of IGEC, a search was made for the implementation of the intellectual level of software and adequate knowledge representation for mobile robots, the construction of heuristic deductive reasoning and decision making.

In accordance with [1-4], it was suggested that the ancient Indian (I) tradition presupposes reliance on the senses and represents contemplation, intuition as research methods and evidence. 
Ancient Greek $(\mathrm{G})$ tradition is based on logical conclusions, rational reasoning and uses the methods of the natural sciences. Ancient Egyptian (E) tradition presupposes reliance on authority: its affirmations are by all means the truth, and the exact execution of installations is the main subject of the method. Ancient Chinese (C) tradition relies on careful execution of a large number of small operations. Its method is numerous calculations-manipulations, which are typical for modern computer technology. The integrated application and integration of all approaches led to the creation of the so-called IGEC technology, which is a combination of conventionally designated "ancient Indo-Greco-Egyptian-Chinese" traditions and methods of constructing evidence. The specific original methods of each approach (each tradition) and examples of the applications, making informal knowledge computational (it's contained in [2-4]).

Using this analogy, a special four-dimensional cube was introduced in [4] to more accurately characterise the work with all four IGEC approaches, and Pentalogics was used to study its two-dimensional faces (see Fig.3).

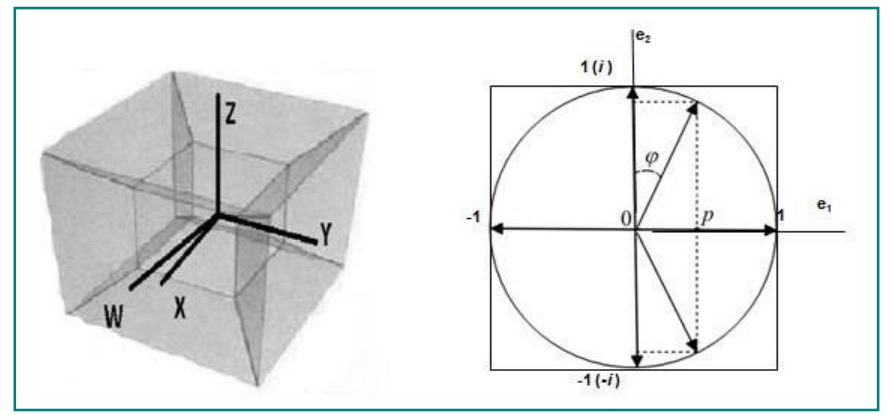

Fig. 3. The four-dimensional cube for IGEC technology and the plane of expert assessments in Pentalogics

The vertices of the cube are numbered by natural numbers: $(1),(2), \ldots,(16)$ and have the following coordinates: $(1)=$ $\langle-1,-1,-1,-1\rangle,(2)=\langle 1,-1,-1,-1\rangle, \ldots,(16)=\langle 1,1,1,1\rangle$. Let's interpret the axes of the cube's coordinates as follows: OX (length) - Ancient Greek measurement, OY (width) - Ancient Egyptian, OZ (height) - Ancient Chinese, OW (fourth axis) - Ancient Indian. Thus, each of the 4 coordinates changes in the range [-1..0] U [0..1], which shows the "success" of the corresponding approach for a task, that is characterised by a point or region of this cube. Pentalogics was proposed in [3] as a separate apparatus with the idea of formalising pairs of expert estimates in the ranges [-1..0] $\mathrm{U}$ [0..1], interpreted as the coordinate on (complex) plane as follows. The estimation along the abscissa axis corresponds to the level of knowledge: "I know-false, I do not know, I know-true", and the ordinate axis shows the so-called imaginary component of the assessment: "I do not believe, uncertainty, I believe."

Let us now consider the issues of the appearance of contradictions and their overcoming. As noted above, the results of each of the approaches for specific tasks may somehow contradict each other. Note that in this case we are not talking about logical contradictions and ways to overcome them, considered in [1-3], but "information", having different nature: according to one source of information (indicating the degree of reliability), the fact $\mathrm{P}$ holds, and according to another not-P. To clarify and overcome such information contradictions, we extend Pentalogics to the two-dimensional faces of the four-dimensional cube in the following way. Fix some two (for example, putting them equal to 0 from 4 coordinates and get a square for the estimation of the role of the two remaining approaches - Fig. 3 (right-hand side). In this way, analogous to the individual study of each of the 4 approaches, a method of investigating 6 pairs of their combinations appears, which can be done with the help of a Pentalogics apparatus. By interpreting the axes of coordinates with the values, of the corresponding approaches, we obtain specific Pentalogics for the formalisation of expert estimates. It is easy to verify that, up to a permutation of the coordinate axes, we have 6 pairs of Pentalogics, which we denote as follows: EG (Egyptian-Greek), EC (Egyptian-Chinese), IG (Indo-Greek), IC (Indo-Chinese), GI (Greek-Indian), GC (GreekChinese). So, if, for example, OX is an Ancient Egyptian dimension, and OY is Ancient Greek, then we have the socalled EG-Pentalogic, in which, in particular, the relations between the two approaches discussed above can be formalised. Here, the level of scientific knowledge can be compared on the abscissa axis: "science knows-lie, science does not know, science knows-true", and evaluation on the ordinate axis is an authoritative component of the assessment: "authority does not approve, uncertainty, authority approves").

\section{Explanations to the industrial application of IGEC technology.}

In the process of application of IGEC-technology to actual practical tasks there is a structuring, specification and clarification of each of its approaches, their coordination and development. At the same time, each of the four approaches of the IGEC using a logical device is "tuned" to specific areas of the subject area, and the use of Pentalogics makes it possible to detail their "inter-traditional junctions" and combine the results into a single whole. So, it seems to be promising application of IGEC in the process of designing, creating and maintaining a management system for the workshop, working on the technology "Industry 4.0". In this task, the most important problems are the synthesis, testing, designing a program control for this equipment, as well as formalisation and construction of a suitable imitation models. We indicate the ways of the solution with the use of IGEC-technology. 
Logical formalisation and algorithms allow constructive, correct and complete solutions written in the language of production rules, Boolean functions, together with their deductive proofs. The visualisation of results, the construction of a strategy maps, allows you to structure and understand arrays of different types of information. Building Boolean functions leads to unification of measurement results, which is important for compatibility with other digital technologies. Logical scaling of the results, performed, for example, by means of propositional logic, and visualisation (including in terms of tasks terms), allows you to drill down into large amounts of data and bring it to a clear understanding: expert, logical, intuitive. The coordinated application of all IGEC approaches, together with the use of Pentalogics, helps diagnose and eliminate errors, smooth out contradictions ("see one" and "the program gives out another", the program conflicts with the expert, the results of the program are not supported by evidence, etc.) and iteratively refine the concepts in all planes on the axes of traditional approaches.

On the I-approach, visualisation of the main processes is carried out, implemented on a special layout. According to the G-approach, we distinguish the key "positions" in the state of this equipment - normal (effective), to which one should strive; dangerous, which must be avoided; and also describe illegal (impossible) positions. Applying the E-approach, we refine and supplement the concepts with the help of expert technologists. Starting the C-approach, we obtain estimates of other (not key) positions of interest to us (illegal, legal-normal, legal-dangerous). At the stage of verification and presentation of results, we again apply all approaches (we especially note the generation of product rules <situation, action>) by analogy with the application of IGEC to chess endings. Finally, with the help of Pentalogics, we identify and eliminate contradictions in all 6 planes of traditional approaches. So, for example, the discrepancies in the results of inference, computational experiments and the conclusions of a technologist are studied with the help of Pentalogics in the GC, EG and EC planes. And the addition of visualization and modelling on the layout allows you to eliminate the contradictions in the IE, IR and GI planes. The implementation of such a software-hardware production control system based on IGEC technology is in the prototyping stage.

When the work of the workshop is formalised, for each numbered object in the diagram in Fig. 1, sets of Boolean variables that characterise their state are introduced. Thus the additional numerical variables $Z_{i}$ are introduced, which allow diagnosing pipe states in the production scheme and object connections. The entered variables and their specific values allow us to describe the current state of the system, called configurations or positions, via the function $\mathrm{f}(\mathrm{x})$, where $\mathrm{x}$ is a set of values for variables. For the class of configurations in which the workshop works properly ("successful" configurations), we assume that $\mathrm{f}(\mathrm{x})=1$. For the class of configurations in which the workshop is off, we assume that $\mathrm{f}$ $(x)=0$. If the shop fails during it's functioning, then we assume, that $f(x)=-1$. Thus, at this stage, the functioning of the workshop is represented by three-valued logic, which can be translated into special Boolean algebra or propositional logic by special translation. One of the principal problems is the large number of Boolean variables used in the model of this complex industrial production, which generates a significant amount of configurations to be studied and classified. To overcome this problem, special software is used and adapted to work with a big-data [12].

Problems of the second type include the elimination of contradictions, that have a non-logical, but "versatile" nature, which were described above, when describing the four-dimensional space of IGEC technology. The main tasks are: coordination of the data from different sensors, harmonisation of the recommendations of the technologist and automatic programs, harmonisation of various equipment standards, harmonisation of classical and modern service traditions, etc. All this is displayed on the corresponding IGEC-plane in order to understand the reason for such an "induced" contradiction and to eliminate it.

It is envisaged to enter data from a system of sensors and feedbacks. that control the operation of all actuators with logical analysis and identify the incorrect progress of production, which ensures the prediction of the state of assemblies and units in order to prevent disruption of the technological cycle.

The developed system for control the production cycle includes an interface that provides round-the-clock remote access to it, through cloud services and the Internet, for the purpose of remote monitoring, testing and operational modernisation of the technological processes. The interface allows you to create and merge several production processes into a single system.

The described production includes a set of fairly common technological operations, which make it possible to clearly illustrate the application of the principles of the concept Industry-4.0.

The work was carried out with the partial financial support of the RFBR, projects 16-07-00935, 16-07-01264, 16-0700811 .

\section{References}

[1] Kirilchenko AA, Pryanichnikov VE, Rogozin K.V. Limits of reliability and reliability of evidence. Skepticism in mathematics, functions, traditions // Information-measuring and control systems. - 2013. Vol. 11, No. 4. - P. 57-65.

[2] Pryanichnikov V.E., Katalinic B., Kirilchenko A.A., Khelemendik R.V., Kuvshinov S.V., Vician D., Uglesic A. New Creative Educational Technologies for Inter-university Network // 25th DAAAM International Symposium on Intelligent Manufacturing and Automation. 2014. / Procedia Engineering. 2015. Vol. 100, - P.259-268. - URL: http://www.sciencedirect.com/science/article/pii/S1877705815003938 
[3] Pryanichnikov VE, Bieleich T., Vitsan D., Catalinich B., Kirsanov KB, Kuvshinov SV, Marzanov Yu.S., Poduraev Yu.V., Khelemendik RV, Prysev EA, Ugleshik A., Kharin K.V. Development of educational technologies and a network of associated laboratories-robotariums // Information-measuring and control systems. - 2015. - T. 13, No. 7. - C.7-25.

[4] Pryanichnikov VE, Khelemendik R.V. Information technology IGEC and Pentalogics / / Computer Science and Information Technology: Materials of International. Scientific Conf. - Saratov: The publication Centre "Science", 2016, pp. 331-333.

[5] Uspensky V.A. Seven Reflections on the Themes of the Philosophy of Mathematics // Regularities in the Development of Modern Mathematics, Moscow: Nauka, 1987, pp. 106-155.

[6] Frank S.L. Religion and Science // Religion, Philosophy and Science. 1953. - No. 1. - P. 1-26.

[7] Gorbunov-Posadov, M.M. Painless development of the program // Open systems. - 1996. - № 4. - P. 65-70. - URL: http://www.keldysh.ru/gorbunov/evolution.htm

[8] Frankl V. Man in search of meaning. Collection. / Translated into English. - Moscow: Progress, 1990. - 368p.

[9] Chess endings: Pawn. - 2 ed., Ext. / Ed. Yu.L. Averbakh. - Moscow: Physical training and sports, 1983. - 303s.

[10] Khelemendik R.V. On the solution of chess positions with the help of formulas for the logic of branching time, Izv. Sarat. un-ta. New Ser. Ser. Mathematics. Mechanics. Computer science. - 2011. T. 11., Iss. 1. P. 111-121.

[11] Endgame Nalimov Tablebases Online [Electronic resource]. URL: http://chessok.com/?page_id=361 (circulation date April 10, 2017).

[12] Khelemendik R.V. SFECalc: Program for constructing minimised circuits from functional elements, analysing them and synthesising them with specified parameters using large Boolean formulas / Certificate of official registration of the computer program No. 2013615516, registered in the computer program registry. 